\title{
El laberinto del modernismo y la vanguardia en la Península Ibérica: dramatis personae luso-español
}

\author{
Antonio SÁEz Delgado \\ Escola de Ciências Sociais, Centro de Estudos Comparatistas \\ Universidade de Évora \\ asaezdelgado@gmail.com
}

\begin{abstract}
RESUMEN
Es posible interpretar el tiempo del modernismo y la vanguardia en el espacio ibérico como un continuum, sin cortes estéticos radicales, que configura una visión múltiple y heterogénea de la modernidad peninsular, observando los puntos de contacto existentes entre escritores, movimientos, escuelas y tendencias desde una perspectiva supranacional, como un polisistema activo y dinámico en el que se alternan las literaturas hegemónicas y periféricas.
\end{abstract}

Palabras clave: Comparatismo ibérico, modernismo, vanguardia.

The Labyrinth of Modernism and Avant-garde in the Iberian Peninsula: Portuguese-Spanish Dramatis Personae

\begin{abstract}
The period of modernism and the avant-garde in the Iberian region may be interpreted as a continuum, with no radical aesthetic divide, shaping a multiple and heterogeneous view of modernity in the peninsula, allowing to observe the contact elements among writers, literary movements, schools and trends, from a supranational perspective, as an active and dynamic polisystem where hegemonic and fringe (peripheral) literatures alternate.
\end{abstract}

Keywords: Iberian comparative studies, modernism, avant-garde.

\section{Introducción}

Un siglo después de la aparición y del asentamiento de los ismos europeos, se hace necesario acercarnos a su presencia en el espacio ibérico desde una perspectiva integradora, que intente alcanzar una visión rigurosa sin la excesiva rigidez de los planteamientos que analizan un fenómeno ideológico y estético aislado del contex- 
to y del proceso en que surge. Desde esta posición, que escapa a los límites estrechos de las literaturas nacionales y nos hace, con las herramientas del comparatismo, entender las literaturas ibéricas como un polisistema literario, es posible acertar a vislumbrar marcas visibles que relacionan identidad y territorio en la Península Ibérica en una visión transnacional, con un mapa múltiple y variable en el que el papel hegemónico fluctúa a ambos lados de la principal frontera que divide en dos el territorio ibérico.

Así, es posible acertar a construir un itinerario que nos lleva desde el modernismo a la vanguardia histórica como un periplo por un espacio múltiple y dinámico en el que se ponen en escena las tensiones entre centro y periferia propias del caso singular de la Península Ibérica, y que acaba convirtiéndola en una geografía heterogénea y multicultural que funciona como un rico sistema de vasos comunicantes. Esta perspectiva nos permite profundizar en la posibilidad de entender el tiempo del asentamiento de la modernidad en el espacio ibérico no solo como una secuencia de carácter histórico, y por lo tanto susceptible de ser estudiada con las estrategias propias de la historiografía literaria, sino, en paralelo, con las puertas abiertas a una interpretación de los hechos basada en la singularidad del espacio en que se desarrollan como marco plural de identidades y culturas en relación o conflicto.

Se hace necesaria, en definitiva, una perspectiva que consiga integrar en un solo diálogo activo las voces de las diferentes literaturas de nuestro contexto, con la seguridad de que todas ellas cumplen un papel esencial en la construcción del espacio ibérico modernista, con frecuencia olvidado por la supremacía de la que la literatura española (entiéndase, en castellano) ha gozado en una visión tradicional del problema, que tantas veces, incluso, ha obviado la existencia -y no digamos la posibilidad- de ampliar el foco de atención a los contactos establecidos con la literatura portuguesa, la catalana, la gallega, etc. Esta multiplicidad lingüística y cultural ha sido con frecuencia enterrada en una visión historiográfica que concedía un lugar preeminente a la «literatura nacional española», con la evidente problematización implícita en ese sintagma, que a las diferentes implicaciones del término «nacional» (Guillén 1998: 299) une una connotación ideológica (Valdés 2004: 15-17) fundamental en el caso que nos ocupa. Y aunque la literatura portuguesa ha ocupado, en buena medida, un lugar paralelo al de la literatura española, con un grado de autonomía y singularización historiográfica irrefutable, tampoco ha formado parte de la tradición crítica peninsular la voluntad de situar en el mismo tablero de juego las piezas de estos dos sistemas nacionales mayores para observar la permeabilización de sus frutos. El propio R. Menéndez Pidal ya denunció, en cierto modo, esta misma situación desde el prólogo de la Historia general de las literaturas hispánicas, donde afirma sin contemplaciones que «lo que aún más hace perder el interés al estudio de la literatura española en el concierto de las demás es el historizarla sin relacionarla debidamente con los hechos de las literaturas extranjeras» (Menéndez Pidal 1949: XLIII).

Desde esta visión hasta la necesidad actual de una perspectiva transnacional, y no solo hispanista, de los estudios ibéricos (Resina 2009), ha transcurrido más de medio siglo en el que las literaturas periféricas han reivindicado su papel en la «ten- 
sión dialéctica» (Gutiérrez García 2004) existente entre las literaturas peninsulares. Más de medio siglo que nos deja una conclusión evidente: a la siempre compleja relación entre identidad e ideología en el contexto de las diferentes literaturas nacionales de la Península Ibérica, debe añadirse ahora una cuestión que se alza de forma indeleble sobre el mapa ibérico: la literatura, la obra literaria, es el texto en cuanto producción, por supuesto, pero también el texto en cuanto recepción, como elemento de transmisión de valores culturales susceptibles de ser asimilados o rechazados en el entorno cultural de llegada. Las literaturas de la Península Ibérica entendidas como un campo de flujos y reflujos estético-ideológicos no pueden restringirse a una concepción meramente genética, sino que deben ser entendidas como un campo plural y múltiple de oportunidades de relación en un contexto geográfico y temporal especialmente propicio, basado en la idea de que la obra literaria es una estructura dinámica.

\section{Pluriverso ibérico}

Ciertamente, situándonos en el campo de las literaturas de la Península Ibérica en el tiempo del modernismo y la vanguardia histórica, no es difícil afirmar, con C. Guillén, que «los componentes fundamentales de la historiografía literaria, es decir, las unidades extensas, como los periodos, las corrientes, las escuelas o los movimientos [...] no suelen reducirse a ámbitos nacionales» (Guillén 1985: 333). $\mathrm{Si}$, además, como es nuestro propósito, prestamos una atención especial a las relaciones entre las literaturas española, o de expresión castellana, y portuguesa en el periodo mencionado -aunque sin desatender ciertas noticias sobre las letras catalanas y gallegas-, estas palabras cobran una luz singular, y nos obligan a integrar dos disciplinas, como la Historia Literaria y la Literatura Comparada, cuya complementariedad se hace imprescindible en el caso que nos ocupa. Así, conseguiremos pasar de una visión de sistema único y monolítico (o de la suma de varios de ellos) a un polisistema dinámico y en constante movimiento, que integra estructuras que van mutando y transformándose muchas veces a partir de un principio de oposiciones internas. Un polisistema en el que, en definitiva, podamos estudiar, en paralelo al universo genético de los autores, el pluriverso de los receptores, sean estos traductores, mediadores o transformadores que sirven de puente entre literaturas en contacto.

En el caso concreto de la literatura española, probablemente el conflicto terminológico y conceptual creado alrededor del término «modernismo» haya ayudado poco a esclarecer con el debido rigor el curso de los contactos establecidos en las tres primeras décadas del siglo XX con otras literaturas, al no acabar de ser entendido como sinónimo del continuum de la modernidad española (Mainer 2000: 348). Por su parte -planteamiento que, a su vez, se ha dado en el caso de las letras catalanas tras abordarse la revisión del alcance del Modernisme (vid. Marfany 1974, 1975)-, las distancias terminológicas del mismo término a propósito de las literaturas española y portuguesa son conocidas (Silvestre 2001) y han generado una problemática que se ve iluminada si aplicamos a la literatura española las palabras 
que Jorge de Sena (1919-1978) utilizó para diagnosticar la portuguesa del mismo periodo:

Ao longo da primeira metade do século XX, duas linhas fundamentais são o que dialecticamente compõe o Modernismo: o que se pode chamar pós-simbolismo (continuando, em experiências e apuramentos pessoais, o que o simbolismo experimentara) e o vanguardismo (que parece ser, e de certo modo é um corte iconoclástico com as tradições literárias e estéticas em geral). (Sena 1978: 85)

Esas «líneas» a las que se refiere Jorge de Sena, que podríamos identificar en la historia de la literatura española, respectivamente, con el modernismo (y postmodernismo) y la vanguardia histórica (ultraísmo y creacionismo), sin embargo, han sido demasiadas veces interpretadas en el contexto español como rayas de separación que marcan momentos diferentes y diferenciados -con un corte más o menos brusco- de un recorrido que, a fin de cuentas, podría ser el mismo, y que desemboca en la asunción de la vanguardia como tradición y de la tradición como vanguardia que encarna la Generación del 27, como parte final de un proceso de asentamiento de la modernidad que podemos entender como un continuum simultáneo y no como un conglomerado de sucesivos movimientos, escuelas y generaciones que se superponen en el tiempo. Esa es, en definitiva, la lúcida propuesta de Jorge de Sena para la literatura portuguesa, dos líneas que conviven dialécticamente durante décadas (en convivencia pacífica o en pugna estética e ideológica) para construir el proceso múltiple y heterogéneo del modernismo, y que nos sirve para iluminar la realidad de las letras españolas del momento y poder, por fin, situar ambos sistemas nacionales en un mismo campo de juego.

En efecto, es posible trazar desde la historiografía líneas paralelas que cruzan de forma análoga las literaturas española y portuguesa del tiempo modernista y la vanguardia, y que nos permiten acercarnos a una cronología común que podríamos cifrar en tres momentos fundamentales (Sáez Delgado 2010: 29-43), teniendo siempre en cuenta que los tres constituyen un mismo sendero en el que sus frutos se superponen: a) el momento del simbolismo / modernismo, que podríamos encuadrar entre 1890 y 1915 ; b) el tiempo de la vanguardia histórica, entre 1915 y 1927; c) el momento de la «segunda vanguardia», desde 1927 hasta el estallido de la guerra civil española. Desde esta perspectiva integradora, que traza líneas casi invisibles en el curso de un proceso que debe ser entendido como un continuum, es posible situar movimientos, escuelas y generaciones en diálogo abierto, con la finalidad de encontrar los elementos de contacto existentes entre los escritores de los dos países.

Así sucede, exactamente, en el primer estadio mencionado, el del simbolismo / modernismo, donde no se hace difícil localizar puentes de contacto entre los simbolistas y saudosistas portugueses con los modernistas y noventayochistas españoles. Las diferencias estéticas e ideológicas existentes entre los seguidores de Rubén Darío y Miguel de Unamuno en la literatura española son, en no pocos aspectos, semejantes a las que encontramos entre los poetas «nefelibatas» que seguían a Eugénio de Castro y los saudosistas que se reunían alrededor de Teixeira de Pascoaes (1877-1952). Del mismo modo, ya en el segundo estadio, las preocupaciones e 
inquietudes de los jóvenes escritores reunidos alrededor de la revista Orpheu (1915) y el primer modernismo portugués - con papel destacado para Fernando Pessoa (1888-1935), Mário de Sá-Carneiro (1890-1916) y José de Almada Negreiros (18931970) - son equiparables a las de los poetas ultraístas y creacionistas, de la misma forma que la Generación del 27, finalmente, ofrece similitudes con el segundo modernismo portugués, vinculado a la revista de Coimbra Presença (1927-1940).

Sin embargo, y aquí radica la riqueza de la visión múltiple y dinámica que proponemos, el hecho de que podamos hacer coincidir las balizas cronológicas fundamentales de estos tres momentos, así como el que podamos establecer parámetros solventes de semejanzas ideológicas y estéticas entre los movimientos, escuelas y generaciones que los protagonizan, no significa en absoluto que los caminos trazados por ambas literaturas fuesen comparables a la hora de analizar sus frutos. De hecho, la realidad nos indica que, de los tres momentos referidos, el más importante en la literatura portuguesa, desde el punto de vista del canon, es el segundo, el del primer modernismo, mientras que en la literatura española ocurre exactamente al contrario, destacando el primero (la Generación del 98) y el tercero (la Generación del 27) sobre el momento histórico de la vanguardia. Es decir, la imagen que proyecta esta situación es la de dos piezas de puzzle que encajan a la perfección, alternando a ambos lados de la frontera los momentos de hegemonía en el polisistema ibérico. Esta situación, de hecho, tendrá mucho que ver con el establecimiento y revisión del canon literario de ambos países, al que deberemos acercarnos como una concepción dinámica (Mainer 1998: 272) que representa las líneas de fuerza de una literatura nacional en un momento histórico determinado. Pero es importante también tener en cuenta que, a pesar de lo anteriormente dicho, no podemos caer en la tentación de pensar que los elementos menos poderosos (desde el punto de vista de la producción en forma de libro y del propio canon) de esta cadena merecen una atención menos consistente, del mismo modo que no debemos ceder a la posibilidad de acercarnos al panorama propuesto prestando tan solo atención a los nombres más importantes que encierra cada uno de sus segmentos. La visión de continuum que defendemos hace incomprensible defender el valor del primer modernismo portugués encabezado por Fernando Pessoa sin colocar en un lugar bien visible el papel desempeñado por saudosistas y simbolistas, por ejemplo, del mismo modo que difícilmente podremos acercarnos a la Generación del 27 y al valor de su poesía sin conceder un lugar también destacado a los poetas del ultraísmo y la vanguardia histórica española. Esta perspectiva de trabajo nos obliga, sin duda, a elaborar un mapa dinámico y múltiple, en el que, junto a un puñado de autores de gran dimensión, conviva también un grupo notable de escritores, traductores y mediadores que con frecuencia no consiguieron sobrevivir a su tiempo, pero que dotan de un relieve fundamental al territorio que nos ocupa.

Uno de los casos tipológicamente más significativos en este panorama es el del poeta de Coimbra Eugénio de Castro, que publica en 1890 el libro Oaristos, que podemos considerar como la primera obra asumidamente simbolista publicada en la Península Ibérica (Sáez Delgado 2008: 13-35). Castro, que no forma parte en la actualidad del canon de la literatura portuguesa del siglo XX, fue sin embargo uno de los autores más importantes de su tiempo a escala internacional, como lo demues- 
tran tanto su amplio epistolario hispánico (Álvarez y Sáez Delgado 2007) como su presencia en Los raros, donde Rubén Darío reivindica su figura como el poeta más importante del momento, junto a Gabriele D’Annunzio. Eugénio de Castro gozó en España de un prestigio incomparable con el de ningún otro autor luso en toda la primera mitad del siglo XX, y solo equiparable al que alcanza Fernando Pessoa en el último cuarto de la centuria. De hecho, su obra fue admirada tanto por Darío y el modernista Francisco Villaespesa (1877-1936) como por Miguel de Unamuno, convirtiéndose en el autor luso más presente en las revistas españolas -La vida literaria (1899), Revista Latina (1907-1908), Renacimiento latino (f. 1905), Prometeo (1908-1912), Los Quijotes (1915-1918), Cervantes (1916-1920) o Grecia (19181920) - y del que más traducciones se realizaron, tanto en antologías -Del cercado ajeno, en 1907, de Enrique Díez-Canedo (1879-1944) (Díez-Canedo 1907); Atlàntiques, de Ignasi de Ribera i Rovira (1913) y con prólogo firmado por Joan Maragall, de marcado corte iberista; o Las cien mejores poesías líricas de la lengua portuguesa, de Fernando Maristany (1918)- como a través de obras individuales.

Entre estas, destacan títulos y traductores como Constanza (traducción de Francisco Maldonado y prólogo de Unamuno, 1913), El rey Galaor (traducido por Juan González Olmedilla en 1913), Salomé (traducido por Francisco Villaespesa en 1914) o el primer volumen de sus Obras en castellano (en traducción de González Olmedilla de 1922), donde se recogían Oaristos y Horas. Tan importante fue su recepción en España que varios escritores, entre los cuales el modernista-ultraísta Rogelio Buendía (1891-1969) (en las páginas de Lusitania, 1920) y el también periodista César González Ruano (1903-1965) (desde Un español en Portugal, 1928), visitan al poeta en Coimbra y narran sus encuentros (Sáez Delgado 2012: 39-54), algo que también hará, en 1931, Mauricio Bacarisse (1895-1931) en las páginas de su novela Los terribles amores de Agliberto y Celedonia, donde la pareja de protagonistas visita en una ciudad sin nombre que es Coimbra al «gran poeta parnasiano» (Sáez Delgado 2012: 117-136). Estas circunstancias convierten a Eugénio de Castro en el poeta que más y mejor permeabiliza el tejido de la literatura española en las primeras décadas del siglo XX, cuando su simbolismo fue admirado y seguido por numerosos autores mayores y menores, traductores y mediadores que harán una importante contribución a la expansión de su obra y a la recepción e integración de la misma entre los autores castellanos y catalanes, pues tanto Ignasi Ribera i Rovira como Fernando Maristany también divulgaron su poesía en el contexto de la literatura catalana.

Fernando Maristany es también protagonista directo y de primera línea en el proceso de recepción de otro de los grandes poetas portugueses del momento, como es Teixeira de Pascoaes, apóstol del saudosismo (Sáez Delgado 2008: 37-76), que conoció tres libros traducidos en España: Tierra prohibida (traducción de Valentín de Pedro, 1920), Regreso al paraíso (traducción de Fernando Maristany y prólogo de Leonardo Coimbra, 1922?) y la antología Pascoaes (Maristany 1920?). El de Amarante, como Eugénio de Castro, fue amigo de Miguel de Unamuno, que prologó la traducción española de San Pablo y le dedicó palabras generosas en Por tierras de Portugal y de España, en 1911.

La acogida que la poesía de Teixeira de Pascoaes y su propuesta saudosista tuvo en España y, en concreto, en Cataluña y Galicia, resultó tan generosa que fue invi- 
tado a pronunciar una serie de conferencias en Barcelona en 1918, reunidas poco tiempo después en su libro Os poetas lusíadas. Allí lo recibió el círculo de Eugeni d'Ors (1881-1954) (quien le dedica unas interesantes páginas en el Nuevo glosario), como también lo hicieron el mencionado Maristany (para quien Pascoaes prologa su libro En el $a z u l$ ) e Ignasi Ribera i Rovira. De hecho, en la antología de poesía portuguesa que publica Maristany en 1918 Teixeira de Pascoaes es el poeta de la historia de la lírica lusa que aparece con mayor número de poemas, y al que se dedica la parte más importante del interesantísimo prólogo que firma Ignasi Ribera i Rovira, donde traza significativas líneas de contacto y de sintonía espiritual entre el saudosismo portugués y la «añoranza» o «enyor» catalán. Esta circunstancia, de hecho, ya había sido de alguna forma manifiesta un año antes, en 1917, por Andrés GonzálezBlanco (1886-1924), quien publica en la revista Estvdio (1913-1920) de Barcelona un artículo en el que afirma que «la saudade es de hecho, como palabra, una creación lusitana; mas el sentimiento que informa esa palabra es patrimonio de todos los pueblos de Iberia» (González-Blanco 1917: 397).

Teixeira de Pascoaes visitó también Madrid, en cuya Residencia de Estudiantes pronuncia una conferencia en 1922 titulada «D. Quixote e a Saudade», y viajará a Galicia, que considera una parte de su patria espiritual, donde alcanza una notoriedad importante gracias a los círculos de las revistas A nossa terra (fundada en 1907), Alfar (1920-1927) y Nós (1920-1936) (Cameirão 2010b). Su poesía de corte trascendente fue acogida, así, en varios contextos de la Península Ibérica como una posible fórmula estética frente a los vaivenes esteticistas del modernismo hispánico, con un profundo poso nacionalista que la aproxima, en no pocos aspectos, a los planteamientos ideológicos de los hombres del 98, pero siempre con una visión plural y dialogante del territorio ibérico, sobre el que se cuestiona en textos fundamentales como «A alma ibérica», con la base del posible encuentro entre los elementos simbólicos representados por la saudade y don Quijote.

Entre Eugénio de Castro y Teixeira de Pascoaes, dibujando un triángulo preciso y actuando como eje fundamental en la recepción de ambos autores en España, destaca la figura del bilbaíno Miguel de Unamuno, convertido en las primeras décadas del siglo XX en una de las figuras más prestigiosas de la vida intelectual peninsular. $\mathrm{Su}$ vocación iberista es incontestable, como lo demuestran los más de trescientos libros en portugués que atesoró en su biblioteca y los más de cincuenta artículos y una docena de poemas que dedicó al país luso (Marcos de Dios 2010). Miguel de Unamuno mantuvo contactos con Guerra Junqueiro (1850-1923), Eugénio de Castro y Teixeira de Pascoaes, con los que compartió, de forma especial, su interés por desarrollar una conciencia estética en la que siempre estuviese presente un poso activo del componente genuino nacional de cada territorio ibérico.

Miguel de Unamuno encontrará en cada uno de estos tres autores un elemento fundamental a la hora de construir su imaginario portugués: en Guerra Junqueiro, el compromiso ético militante y el campo abierto por el concepto de «patria»; en Eugénio de Castro, la esencia cultural portuguesa a través de la lectura de Constanza, y en Teixeira de Pascoaes, el autor con el que mantuvo un contacto más estrecho y fecundo, una dimensión espiritual del pueblo portugués que hasta entonces no había vislumbrado en toda su intensidad. Si a estos elementos le añadimos la conciencia 
trágica que encontró en su contacto con Manuel Laranjeira (1877-1912), el dibujo del Portugal unamuniano alcanza sus claves esenciales. El rector salmantino fue, al mismo tiempo, colaborador activo de la revista portuense A Águia (1910-1932), órgano de la Renascença Portuguesa, vinculada al saudosismo, donde publica, entre otros poemas, el conocido soneto «Portugal», ilustrado por António Carneiro (18721930) en 1911, el mismo año en que aparece Por tierras de Portugal y de España, obra que alcanzó una difusión bastante importante en Portugal, y que llegó a las manos del propio Fernando Pessoa, en cuya biblioteca personal continúa existiendo un ejemplar.

Miguel de Unamuno es, de los tres nombres mayores citados hasta el momento, el mediador más eficaz para favorecer la difusión y recepción de las propuestas estéticas de sus dos amigos portugueses (de Teixeira de Pascoaes, muy en especial) en España, y es también el autor que más escribe sobre el otro país, por mucho que Eugénio de Castro dedicase en 1923 un libro de poemas a asuntos españoles (A Mantilha de Medronhos) y por más que Teixeira de Pascoaes escribiese algunos textos consagrados a su personal visión de Iberia. En esta labor de mediación se vería compensado por Pascoaes, que llevó su nombre a la revista $A$ Águia a inicios de la década de los diez, donde muy probablemente lo encontró Fernando Pessoa, lector y también colaborador de la publicación. A partir de ese instante, el autor de los heterónimos intentó llamar la atención de Miguel de Unamuno a través del envío de la revista Orpheu, a la que Miguel de Unamuno no debió concederle demasiado tiempo ni interés.

Esta relación imposible entre Miguel de Unamuno y Fernando Pessoa, que aunque compartían algunas preocupaciones (con el concepto de patria y la identidad múltiple del sujeto en primera línea) representaban mundos diferentes, es bien conocida, como también lo son los contactos establecidos por Pessoa con los jóvenes poetas ultraístas Adriano del Valle (1895-1957), Rogelio Buendía (su primer traductor, en 1923) e Isaac del Vando-Villar (1890-1960), director de la revista Grecia (Sáez Delgado 2000, 2002, 2011). Fernando Pessoa nunca demostró un apego especial por la cultura española, pero, sin embargo, escribió bastantes fragmentos sobre la realidad ibérica e incluso parece que llegó a idear un libro de título significativo, Ibéria. Introdução a um imperialismo futuro (Pessoa 2012), que se me antoja una pieza imprescindible para comprender la verdadera dimensión del proyecto mesiánico del quinto imperio cultural, sobre el que tanto escribió.

En ese libro Fernando Pessoa se refiere significativamente a España (entendida como Castilla), Cataluña, Galicia y la posibilidad de una civilización ibérica, en la que la cultura se convertiría en el elemento axial de cara al futuro. Este hecho, además de significativo por contrastar con su no demasiado intenso interés por España (circunstancia que parece, por sí misma, suficiente para demostrar que sus fragmentos ibéricos son una parte notable de su amplia preocupación nacionalista, en cuanto que constructora de imperio y civilización a través de la cultura), singulariza la figura de Fernando Pessoa en el contexto de este breve dramatis personae, pues añade un componente paradójico fundamental a su aproximación hispánica. De hecho, el portugués nunca llegó a desvelar la pluralidad de su obra y su «mensaje» a sus corresponsales españoles, y tuvo que conformarse con ver sus primeros textos en España publicados en medios de difusión especialmente periféricos (los diarios 
andaluces La Provincia, de Huelva, y La Unión, de Sevilla), sin contar nunca con el apoyo ni la mediación de un autor español de gran dimensión.

Este detalle, el desequilibrio entre autores y mediadores en el contexto ibérico, es especialmente significativo y marcará el curso de las relaciones de recepción y permeabilización estética. Fernando Pessoa no contó con Miguel de Unamuno como mediador, como tampoco pudo contar, a pesar de haberse cruzado en algún café lisboeta, con Ramón Gómez de la Serna, el escritor vinculado a la vanguardia española que estuvo más cerca de Portugal y sus escritores (no en vano, se construyó una casa en Estoril a principios de los años veinte) y con el que compartió las páginas de la revista lisboeta Contemporânea (1922-1926).

Ramón escribió sobre Portugal en Pombo (1918) y en La sagrada cripta de Pombo (1924), y ambientó por entero en las proximidades de la capital portuguesa su novela La Quinta de Palmyra (1923). No trató en persona con Pessoa, pero sí lo hizo ampliamente con José de Almada Negreiros, cuya estancia en Madrid entre 1927 y 1932 tuvo mucho que ver con la sintonía del creador de la greguería con el artista plástico y escritor portugués, y también con António Ferro (1895-1956), otro autor del círculo pessoano, en cuyos libros Teoria da Indiferença (1920) y Leviana (1929, con prólogo de Ramón) es bien posible observar la huella greguerística. Y, en paralelo a Ramón, destaca también otra figura fundamental para comprender la verdadera dimensión de su acercamiento a Portugal, como es la de su compañera sentimental Carmen de Burgos, Colombine (1867-1932), que comparte con Ramón su aventura lusa, la amistad con Ana de Castro Osório (1872-1935) y que ambienta en el país vecino algunas de sus novelas cortas, además de publicar en la revista madrileña Cosmópolis, entre 1920 y 1921, un importante conjunto de artículos dedicados a la literatura portuguesa del momento.

Todas estas circunstancias no provocaron, sin embargo, un acercamiento profundo o directo entre los escritores del primer modernismo portugués y de la vanguardia histórica española, más allá de contactos y proyectos personales. El ultraísmo y el creacionismo huidobriano fueron perfectos desconocidos en Portugal más allá de alguna mención muy concreta por parte de António Ferro, del mismo modo que el futurismo portugués de José de Almada Negreiros o el sensacionismo de Álvaro de Campos no formaron parte del universo de lecturas de los más jóvenes escritores españoles, preocupados aún en la década de los años veinte por descubrir a Eugénio de Castro.

Este desentendimiento, no obstante, parece justificado por la diferente naturaleza que la primera vanguardia tuvo en ambos países, con un movimiento español, el ultraísmo, que hizo acopio de tendencias y estéticas para acogerlas a todas bajo el mismo rótulo, con el evidente riesgo conceptual que esa actitud implicaba (como alertó el mismísimo Jorge Luis Borges), y con una realidad en Portugal mucho más diversificada, gracias a la existencia de un futurismo que nunca existió, como tal, en España, y, sobre todo, a las diferentes propuestas estéticas (el sensacionismo, especialmente) nacidas de la mano de Fernando Pessoa. Con este panorama, no es difícil concluir que resulta hasta cierto punto lógico que el ultraísmo fuese, como defendió Guillermo de Torre (1900-1971), más pródigo en gestos que en libros individuales, así como que la pluralidad de la obra pessoana se haya alzado con un lugar 
preeminente en el canon literario portugués del momento. Es decir, con este panorama, no es difícil comprender por qué, como defendíamos antes, el segmento de más interés genético de la vanguardia española es la Generación del 27 (entre cuyas obras figuran, verdaderamente, los frutos del ultraísmo), mientras que en la historia literaria portuguesa será el primer modernismo el elemento más destacado, con Fernando Pessoa a la cabeza, de todo el proceso modernista.

De este modo se configura el mapa al que hacíamos referencia al inicio de estas páginas, un mapa multiforme y heterogéneo desde el punto de vista estético e ideológico, en el que todos sus elementos constituyentes cumplen un papel fundamental en el devenir de la modernidad peninsular. Sin cortes bruscos, con la idea de continuum presidiendo la escena, poniendo en relación no solo movimientos, escuelas y tendencias, sino también el trabajo desarrollado por los grandes autores del momento junto a los autores considerados menores o junto a traductores y mediadores culturales, conseguiremos reconstruir con fidelidad el escenario de aquel tiempo, laberíntico y apasionante como pocos en la Península. Ampliando el objetivo de nuestro estudio a una panorámica ibérica conseguimos releer la literatura modernista y vanguardista desde una perspectiva más neutra, que nos ayuda a situar a cada uno de sus protagonistas en el verdadero lugar que le corresponde en una especie de portátil historia de las literaturas ibéricas comparadas, estableciendo los nexos de relación y los puntos de oposición, las hegemonías y periferias de un tiempo que legó a la historia de las literaturas peninsulares muchos de sus mejores escritores y, al mismo tiempo, muchos de sus más importantes autores menores. 